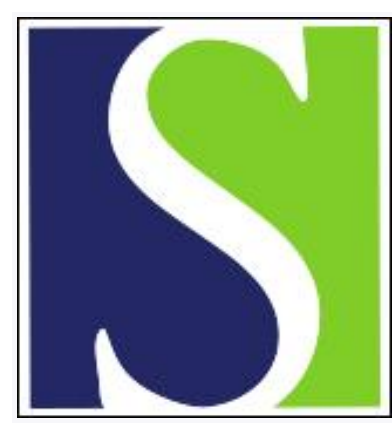

Scand J Work Environ Health 1992;18(1):68-70

https://doi.org/10.5271/sjweh.1606

Issue date: 28 Feb 1992

Cerebral magnetic resonance imaging and cerebral computerized tomography for patients with solvent-induced encephalopathy.

by Leira HL, Myhr G, Nilsen G, Dale LG

Affiliation: Department of Occupational Medicine, University Hospital of Trondheim, Norway.

This article in PubMed: www.ncbi.nlm.nih.gov/pubmed/1553515

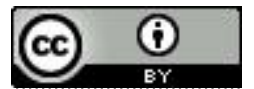




\title{
Cerebral magnetic resonance imaging and cerebral computerized tomography for patients with solvent-induced encephalopathy
}

\author{
by Håkon L Leira, MD, ${ }^{1}$ Gunnar Myhr, MD, ${ }^{2}$ Gunnar Nilsen, MD, ${ }^{2}$ Leif G Dale, MD, ${ }^{3}$
}

\begin{abstract}
LEIRA HL, MYHR G, NILSEN G, DALE LG. Cerebral magnetic resonance imaging and cerebral computerized tomography for patients with solvent-induced encephalopathy. Scand $J$ Work Environ Health 1992;18:68 - 70. To compare the results of cerebral magnetic resonance imaging (MRI) and computerized tomography (CT) for patients with solvent-induced encephalopathy, a parallel study was performed with nine patients. The diagnosis had been given three to five years earlier on the basis of relevant exposure to solvents, findings on neuropsychological tests, a neurological examination, and cerebral CT. There was no progression of pathological lesions from the first to the second CT examination. Four patients with atrophy in the CT examination also had atrophy in the MRI examination. In addition three patients with normal CT findings showed atrophy in the MRI examination. It was concluded that MRI should be the modality of choice whenever diagnostic imaging is necessary in the clinical workout of patients with diagnosed or suspected solvent-induced encephalopathy.
\end{abstract}

Key terms: clinical examination, diagnostic procedure.

Chronic adverse effects of low dose, long-term occupational exposure to organic solvents are still an issue of controversy. There is disagreement concerning both the inductive dose and relevant symptomatology required for the diagnosis. The diagnostic criteria for solvent-induced encephalopathy are based on the length and intensity of the exposure, relevant symptoms, neuropsychological tests indicating mental impairment, and the exclusion of other relevant causes $(1,2)$.

The diagnostic procedures aimed at diagnosing mental impairment usually include neurological and neuropsychological examinations and cerebral computerized tomography (CT). Mental impairment, as measured by these methods, is implicitly assumed to relate to underlying organic brain damage or brain dysfunction (2). Neuropsychological tests seem to be more sensitive than other methods available for the diagnosis of organic cerebral impairment. Cerebral CT has a low sensitivity (3-7). Pathological CT scans are mostly seen for patients with serious disease (8). A pathological CT scan is not a requirement for the diagnosis of solvent-induced encephalopathy $(1,2,7)$.

In order to assess the usefulness of magnetic resonance imaging (MRI) in the diagnosis of patients with suspected solvent-induced encephalopathy, with regard

1 Department of Occupational Medicine, University Hospital of Trondheim, Trondheim, Norway.

2 MR-center, University Hospital of Trondheim, Trondheim, Norway.

3 Department of Radiology, University Hospital of Trondheim, Trondheim, Norway.

Reprint requests to: Dr HL Leira, Department of Occupational Medicine, University Hospital of Trondheim, N-7006 Trondheim, Norway. to brain morphology and possible lesions, a comparative study of MRI and CT was undertaken.

\section{Subjects and methods}

Ten male patients with solvent-induced encephalopathy diagnosed two to four years earlier were eligible for study. One patient withdrew due to claustrophobia. For further details, see table 1. One patient, aged 32 years, had been exposed to solvents for only eight years, but the exposure had been particularly high.

The diagnoses were based on typical symptoms (fatigue, impaired memory and concentration, irritability, and reduced initiative), the occupational history, the duration and degree of solvent exposure, a clinical neurological examination, neuropsychological tests compatible with encephalopathy, and a CT scan. There were no competing causes for encephalopathy, such as alcohol abuse or significant cardiovascular disease. Two patients were still occupationally active; the rest had been granted a permanent disability pension on the basis of the diagnosis. All of the patients had been granted compensation for chronic cerebral impairment from the Occupational Injuries Insurance organization.

Cerebral CT and MRI were performed within a few weeks of each other for eight patients. In one instance the MRI was performed nine months earlier than the last $\mathrm{CT}$ scan.

The first CT scan, performed at the time of the diagnosis, two to four years earlier, was acquired on an EMI 5005 or on a GE 9800 (10-mm thick consecutive slices without intravenous contrast). The same procedure was used for the $\mathrm{CT}$ examination done in parallel with the MRI, this time on a Toshiba TCT $-300 \mathrm{~S}$.

At both $\mathrm{CT}$ examinations the images were routinely evaluated by the two radiologists on duty. For this 
presentation one of the authors (GM) reevaluated the images to rate the atrophy according to an arbitrary scale from 0 to 3,0 representing normal and 3 representing grave atrophy (age being taken into consideration).

The MRI was performed with a 1.5-T superconductive system (Philips Gyroscan S15): transversal SE (spin echo) TR $2100 \mathrm{~ms}$ (heart triggered) and double echo TE 29/90 ms (6.5-mm slice thickness, slice factor 1.1, 14 slices), coronal SE TR/TE $=500 / 30$ (slice thickness $6 \mathrm{~mm}$, slice factor 1.2, perpendicular to the McGregor line, middle slice corresponding to the dorsum sellae, 8 slices) and sagittal SE TR/TE $=500 / 30$ (slice thickness $5 \mathrm{~mm}$, slice factor $1.1,7$ slices).

The MRI examinations were assessed by two radiologists, first separately and then jointly. The results of the CT examinations were unknown to them. Cortical and central atrophy were evaluated on an arbitrary scale from 0 to 3 , as for the CT scans. In addition, atrophy of the vermis cerebelli was evaluated in the sagittal MRI images, according to an arbitrary scale from 0 to 3 . The size of the posterior fossa cisterns was rated as normal or enlarged when all of the MR images were taken into consideration. Routine CT only employs transversal scans.

\section{Results}

There was no significant progression of pathological conditions from the first to the second CT examination.

The main results are compiled in table 1 . All of the patients with brain atrophy in the CT examination revealed atrophy in the MRI examination. However, the MRI examinations showed two additional cases of ventricular atrophy and two additional cases of cortical atrophy. One patient (number 4) with ventricular atrophy grade 2 according to the CT was evaluated as grade 3 with the MRI, and one patient (number 6) with cortical atrophy grade 1 in the CT was rated as grade 2 with the MRI.

One patient (number 4) had changes in white matter similar to those found in subcortical atherosclerotic encephalopathy, and one patient (number 2) had a septum pellucidum cyst. These incidental findings were seen with both the CT and the MRI. In addition the
MRI showed the following features not present in the CT images: atrophy grade 1 of the vermis cerebelli in six patients, five of whom also showed enlargement of the cisterns of the posterior fossa. Only one of these patients (number 5) had significant cerebellar symptoms (dysdiadochokinesia and light ataxia). One patient (number 1) had a focal lesion with high signal intensity in the left thalamus which was interpreted as a lacunar infarction. One patient (number 7) had two small lesions (probable infarctions) in the brain stem and a venous malformation in the right cerebellar hemisphere.

\section{Discussion}

To our knowledge there are no previous publications on MRI and solvent-induced encephalopathy. Comparative studies of MRI and CT in relation to dementia show MRI to be the more sensitive with regard to white matter and grey matter changes and lesions and also with regard to atrophies (9). CT is of minor importance in respect to the diagnosis of solvent-induced encephalopathy $(5,6)$; however, CT may be useful as a means with which to exclude pathological conditions other than atrophy $(6,9)$. Quantitative measurements in the assessment of brain atrophy are not superior to subjective evaluations $(5,7,9)$. Accordingly, we decided to employ a subjective scale for determining the presence and degree of atrophy.

Different radiologists evaluated the CT and the MRI images. This difference could have caused interobserver variation in the grading of cerebral atrophy. The CT images were read by four radiologists and the MRI images by two radiologists, and thus the importance of this source of error was reduced.

The present study lends support to the results of other studies showing MRI to be more sensitive than CT in the diagnosis of atrophy (9). This increased sensitivity of MRI may be due to the multiplanar approach, to the increased sensitivity for discrimination between cerebrospinal fluid and brain matter (9), and to the use of thinner slices in the MRI examinations than in the CT scans. The multiplanar approach also allows a better evaluation of the vermis cerebelli and the posterior fossa cisterns than does a standard CT

Table 1. Cerebral atrophy as judged by computerized tomography (CT) and magnetic resonance imaging (MRI) among patients with solvent-induced encephalopathy. Scale from 0 (normal) to 3 (grave atrophy).

\begin{tabular}{|c|c|c|c|c|c|c|c|}
\hline \multirow{2}{*}{ Patient } & \multirow{2}{*}{$\begin{array}{c}\text { Age } \\
\text { (years) }\end{array}$} & \multirow{2}{*}{$\begin{array}{c}\text { Exposure } \\
\text { (years) }\end{array}$} & \multirow{2}{*}{ Vocation } & \multicolumn{2}{|c|}{ Ventricular atrophy } & \multicolumn{2}{|c|}{ Cortical atrophy } \\
\hline & & & & CT & MRI & CT & MRI \\
\hline 1 & 62 & 37 & Mechanic & 0 & 0 & 0 & 1 \\
\hline 2 & 62 & 39 & Painter & 0 & 1 & 0 & 0 \\
\hline 3 & 62 & 41 & Mechanic & 0 & 0 & 0 & 2 \\
\hline 4 & 62 & 42 & Car Painter & 2 & 3 & 3 & 3 \\
\hline 5 & 60 & 33 & Painter & 0 & 0 & 1 & 1 \\
\hline 6 & 57 & 36 & Painter & 0 & 1 & 1 & 2 \\
\hline 7 & 55 & 34 & Mechanic & 0 & 0 & 1 & 1 \\
\hline 8 & 36 & 20 & Car painter & 0 & 0 & 0 & 0 \\
\hline 9 & 32 & 8 & Greaser & 0 & 0 & 0 & 0 \\
\hline
\end{tabular}


examination. The lack of vermis atrophy in CT is probably due to this fact rather than to a selective toxic effect of organic solvents to different parts of the central nervous system as suggested in a previous report (6). Atrophy of the vermis as present in four of our patients has previously been reported for toluene abusers $(10,11)$, but to our knowledge not for patients with solvent-induced encephalopathy due to occupational exposure.

There are no MRI findings for patients with solventinduced encephalopathy that are pathognomonic to this disease. The high sensitivity of MRI for white matter diseases makes the modality well suited for excluding pathological brain lesions, but may give rise to difficult differential diagnostic considerations due to low specificity.

The MRI findings of this study did not lead to a change of diagnosis for any of the patients. However, they gave reason to claim a higher degree of compensation from the Occupational Injuries Insurance organization for those workers for whom the MRI was positive and the $\mathrm{CT}$ negative.

\section{Concluding remarks}

MRI seems to be more sensitive than CT in the diagnosis of cerebral atrophy among patients with solvent-induced encephalopathy. We suggest that MRI should be the modality of choice whenever diagnostic imaging is deemed necessary in the clinical workout of patients with diagnosed or suspected solvent-induced encephalopathy.

\section{Acknowledgments}

The study was supported by a grant from The Work Environment Fund of the Confederation of Norwegian Business and Industry.

\section{References}

1. Juntunen J. Neurotoxic syndromes in man. In: Institute of Occupational Health. Third advanced course in industrial toxicology. Helsinki: Institute of occupational health, 1978:164-68.

2. World Health Organization (WHO): Organic solvents and the central nervous system. Copenhagen: WHO/ Nordic Council of Ministers, 1985:9-11. (Environmental health series; no 5).

3. Arlien-Søborg P, Bruhn D, Gyldensted C, Melgaard B. Chronic painter syndrome: chronic toxic encephalopathy in house painters. Acta Neurol Scand 1979;60: $149-56$.

4. Gregersen P, Angelsø B, Nielsen TE, Nørgaard B, Uldal C. Neurotoxic effects of organic solvents in exposed workers: an occupational, neuropsychological and neurological investigation. Am J Ind Med 1984;5:210—25.

5. Skjødt T, Svendsen J, Jacobsen EB, Yde S. Værdien af $\mathrm{CT}$ hos patienter med toksisk encefalopati [The value of computed tomographic scanning in toxic encephalopathy]. Ugeskr læger 1986;148:174-7. (English summary).

6. Berstad J, Flekkøy K, Pedersen ON. Encephalopathy and polyneuropathy induced by organic solvents. J Oslo City Hosp 1989;39:81-6.

7. Ørbæk $\mathrm{P}$, Lindgren $\mathrm{M}$, Olivecrona $\mathrm{H}$, Hager-Aronsen B. Computed tomography and psychometric test performances in patients with solvent induced chronic toxic encephalopathy and healthy controls. $\mathrm{Br} \mathrm{J}$ Ind Med 1987;44:175-9.

8. Gyldensted C. Chronic painters' syndrome: toxic encephalopathy with brain atrophy and dementia in professional house painters. In: Wackenheim A, du Bonlay GH, ed. Choices and characteristics in CT. Amsterdam: Kugler publications, 1979:137-40.

9. Johnson KA, Davis KR, Buonanno FS, Brady TJ, Rosen $\mathrm{TJ}$, Growdon JH. Comparison of magnetic resonance and roentgen ray computed tomography in dementia. Arch Neurol 1987;44:1075-80.

10. Lazar RB, Ho SU, Melen O, Daghestani AN. Multifocal central nervous system damage caused by toluene abuse. Neurology 1983;33:1337-40.

11. Boor JW, Hurtig HI. Persistent cerebellar ataxia after exposure to toluene. Ann Neurol 1977;2:440-2.

Received for publication: 3 May 1991 